\title{
Advanced Modeling of Electric Components in Integrated Energy Systems with the TransiEnt Library
}

\author{
Jan-Peter Heckel Christian Becker \\ Institute of Electrical Power and Energy Technology, Hamburg University of Technology, Harburger Schloßstraße 20, \\ 21079 Hamburg, Germany, \{jan.heckel, c. becker\} @tuhh. de
}

\begin{abstract}
Integrated Energy Systems (IES) with the coupling of electricity, gas and heat are assumed to be a suitable concept for future energy systems. For the necessary energy system analysis with respect to dynamics and stability, powerful tools are needed. Such tools should be provided in open toolboxes to make the research more transparent, comprehensive and communicative. It is aimed that scientists collaborate on models for multimodal energy system analysis.

The dynamic simulation is a method that allows to consider transient, non-linear effects and controller design. The TransiEnt Library, developed and established at Hamburg University of Technology (TUHH), offers such a toolbox. Previous versions of the library worked with limited electrical models. In this paper, the extension of the TransiEnt Library with new models, that allow to consider stability effects of the electric grid, is described. To do so, features from Modelica ${ }^{\circledR}$ and its specification are used to create an advanced framework for the electrical part of IES. As a result, IES with interconnected electric grids can be modeled and simulated more precisely.
\end{abstract}

Keywords: Integrated Energy System, Electric Energy System, Load Flow Calculation, Frequency Stability, Voltage Stability, Renewable Energy

\section{Introduction}

The TransiEnt Library (Hamburg University of Technology, 2018) is a Modelica ${ }^{\circledR}$ (Modelica Association 2017) library for the simulation of an IES with high share of renewable energies. The complete library is open-source. The TransiEnt Library was developed within the project TransiEnt.EE (BMWi 03ET4003) as a fundamentally new approach for modeling IES with the method of dynamic simulation. TransiEnt.EE was completed in 2017 and the successor project ResilientEE (BMWi 03ET4048) has been started subsequently. The TransiEnt Library is implemented in the simulation environment Dymola ${ }^{\circledR}$ (Dassault Systèmes, 2018). Within the Transient.EE project the main focus of the entire system modeling was mainly put on heat and gas system representation with a reduced scope of the electrical system part. This paper presents the extension of the TransientEE Library by increased modeling and simulation capabilities concerning dynamic and stability phenomena of meshed electrical grids.

The paper starts with an illustration of the political, technical and organizational background for IES. This includes the goals and already existing results of the two projects mentioned above. The new models' purpose and their level of detail is explained. In section 2, the development and implementation of the new electrical models are introduced and explained in detail. Section 3 contains three implemented example models including the new features. The paper closes with a conclusion and an outlook to further research work.

\subsection{Energy Transition and Integrated Energy Systems}

The motivation of the projects TransiEnt.EE and ResiliEntEE is the persisting transition of the German energy supply known as Energy Transition. It is planned to increase the share of renewable energies in the next decades in order to decrease the carbon dioxide emissions. Furthermore, the nuclear power phase-out was decided after the Fukushima incident in 2011. In the electrical sector, the renewable energies should have a share of at least $80 \%$ in 2050 (German Federal Ministry of Justice und Consumer Protection, 2017).

The fluctuating generation of the renewable energies must be balanced with the volatile consumption. This is only possible by using storage technology. Electric storages are considered, but their power output and storage capacities are limited and costs per kWh energy storage are high. Hence, the idea of IES to couple the sectors electricity, gas and heat is considered. The coupling is performed by primary coupling technologies. These technologies are for example Power-to-Gas (PtG), Combined-Heat-and-Power generation plants (CHP), Combined Cycle Gas Turbine (CCGT), Fuel Cells (FC) but also technologies like gas boilers, gas turbines (GT), electric boilers, known as Power-to-Heat technology. Figure 1 shows an example of an IES with different technologies. 


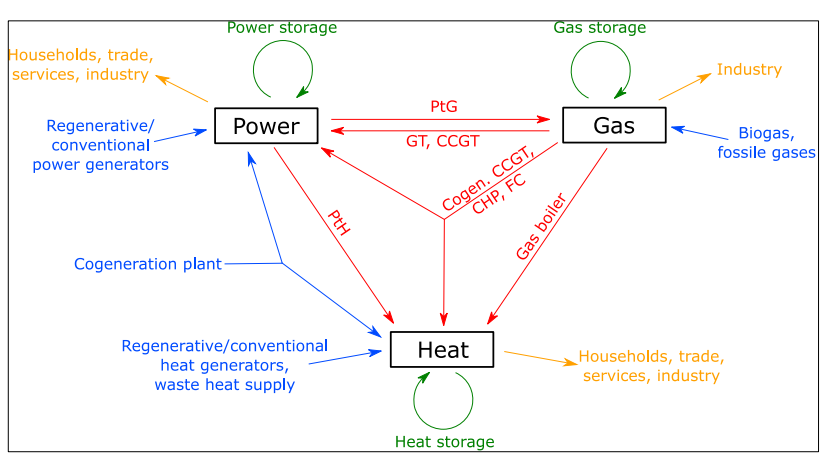

Figure 1. The three sectors with examples of primary coupling technology (red), generation technology (blue), storage technology (green) and civil sectors (yellow)

By multimodal sector coupling, the storage possibilities in the non-electrical sectors can be used for compensating the imbalance between renewable energy generation and consumption. Gas and heat grids themselves are assumed as distributed storages because they can buffer failures much longer than the electric grid. Gas and thermal storage technology are considered to have higher energy capacity, power output and lower costs per kWh energy storage than electric storages.

Nevertheless, future IES must be investigated in a systematic way to estimate their opportunities and risks.

\subsection{Project TransiEnt.EE}

The project TransiEnt.EE investigated innovative possibilities for an efficient energy supply by integrating renewable energies in the existing grid infrastructure. For this, the IES of the city of Hamburg was regarded. The main innovation was the development and use of dynamic simulation means for the entire energy system analysis. Dynamic simulations facilitated to investigate non-linear behavior of components, control strategies and system stability.

It was shown that the sector coupling increases the flexibility of the energy supply. The gained flexibility allows a higher share of renewable energies (Andresen et al, 2017).

\subsection{Project ResiliEntEE}

In the current ResilientEE project, the scope of IES analysis and design is extended to Northern Germany. With this new example region, the topologies of the electric and the gas grid come into the focus of study to a much higher level of detail. The stability of the electric grid coupled with subsystems of the energy sectors and the compliance of the demanded limits for characteristic values are to be investigated.

Finally, the resilience of the IES should be benchmarked. For this benchmark, it is planned to introduce a quantified figure to compare different scenarios. From this analysis, suggestions for further improvement, i. e. an increase of the system's resilience, should be developed. Three predefined scenarios should be regarded within the project: first, a centralized scenario of larger power plants and a centralized control scheme, secondly, a decentralized scenario and thirdly a scenario according to the German grid development plan (Netzentwicklungsplan) 2035.

\subsection{Purpose of Models}

In the frame of the TransiEnt.EE project, the electric grid is modeled as a copper plate. Therefore, the dynamic representation is limited to time-dependent active power balance and system frequency as well as power-frequency-control (Andresen et al, 2015). Furthermore, the TransiEnt Library offers models for unmeshed grids without branches. These models limit the possibilities of electric grid modeling. Standard methods for electric power systems like load flow calculations, angle stability and voltage stability examination are not possible with these models. The modeling approach does not allow to compute voltages and reactive power values.

Hence, it is necessary to develop and implement extended model features that fit into the existing framework of the TransiEnt Library. Components from already existing Modelica Libraries for the modeling of electric power systems are not compatible with this framework. Detailed models for gas- and heatprocesses already exist in the TransiEnt Library. The extension for the electric grid empowers the TransiEnt Library to become a complete and unique toolbox for universal steady-state and dynamic energy system modeling and analysis.

The use of Modelica has many advantages even for the new electrical models. Equations and system structures can be implemented directly. Connectors permit interfaces between sectors and their components and combine the components mathematically to one model.

The main advantage Modelica offers in this context is its modularity. The modularity allows to replace components easily. New features and models can be added by different editors in a simple way.

The TransiEnt Library is designed for energy system analysis performed by energy engineers and users in the energy and power economy. Hence, the electric component models are required to be easy to use for those with different backgrounds as well.

\subsection{Level of Detail}

In complex, dynamic models of energy systems, dynamics of the technologies from different energy sectors are coupled in order to cover their interaction. In general, the electric part of the coupled system has higher dynamics than the processes in the gas and heat sector. Consequently, the risk of a stiff problem occurs. To deal with this risk, models are created in different levels of detail for different time scales which can be replaced by each other. 
In the ResiliEntEE project, systemic concepts for model reduction have to be used. Furthermore, it is deemed to be reasonable to consider only dynamics with time constants above $1 \mathrm{~s}$ in order to avoid stiffness.

\section{Implementation of New Models}

The new models represent interconnected grids with components such as generators, transmission lines and transformers. The basis for this modeling is the complex, quasi-stationary calculation of alternating current networks. The main part of electric grid modeling is the connector which is described in the following paragraph before the components will be introduced.

\subsection{New Connector Complex Power Port}

In the electrical part of the energy system, connectors called Power Ports are used to link several components with each other. In the TransiEnt Library, there are three Power Ports with different levels of detail.

- Active Power Port

- Apparent Power Port

- Complex Power Port

The three Power Ports only regard one phase of the three-phase system assuming this to be symmetric. The Active Power Port is the connector developed and used in the TransiEnt.EE project. Because it just contains active power and frequency, this port can only be used for electric grids modeled as copper plates. The Apparent Power Port is capable of modeling grids without branches, arranged as one line. This is made possible by adding voltage and reactive power to the connector. For calculating the partition of power at branches, another electrical quantity is necessary. The Complex Power Port therefore adds the angle of the complex voltage phasor to the connector quantities.

When regarding the Modelica specification, it becomes clear that the Complex Power Port is overdetermined by one quantity. There should not be more than one potential variable per flow variable. In this case, there are two flow variables, the active and the reactive power, and three potential variables, voltage magnitude, voltage angle and the frequency. The two quantities of the complex voltage can be assigned to the two power flows which all together build the complex representation of the electric network. But the frequency is an additional potential variable which cannot be assigned to a flow variable. Physically, the frequency is not an independent quantity. The frequency is part of the models because of the quasi-stationary approach. Additionally, the frequency is part of the Complex Power Port and hence, an available information in all parts of the electric grid in this modeling approach. Within the model approach for the ResiliEntEE project, it is intended to only have one grid frequency. This is a justified simplification for the grid model of Northern
Germany as it is strongly meshed. For larger networks, different local frequencies must be considered to take care of power oscillations. In section 2.3, it is discussed how to extend the concept to more than one frequency.

To avoid overdetermined Differential Algebraic Equation Systems (DAE) in interconnected electric grids, the procedure mentioned in the Modelica Specification section 9.4 is followed. With this procedure, the Complex Power Port is defined as follows.

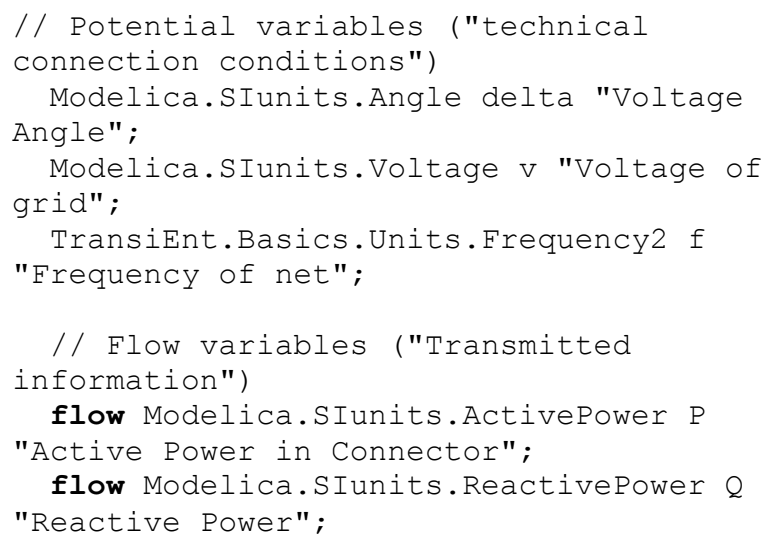

The Type Frequency2 has the function "equalityConstraint" as defined in the specification. The algorithm is:

\section{algorithm}

assert(f1-f2<1e-10,"Equal frequencies");

With this connector, electric grids can be connected via one unipolar connector. This allows the well-known unipolar representation of electric power systems which facilitates the work with the components for users with different backgrounds.

\subsection{Steady-State Components}

Stationary busses for single load flow calculations and components for linking busses are available in the TransiEnt Library as steady-state components. Stationary busses are used as boundaries for different quantities from the Complex Power Port. In electrical power engineering, three kinds of stationary busses are distinguished.

- Slack Bus: Voltage magnitude and angle are fixed; the frequency is given for steady-state calculation and the other quantities are calculated.

- PV Bus: Active power and voltage magnitude are fixed; other quantities are calculated.

- PQ Bus: Active and reactive power are fixed; other quantities are calculated.

The Slack Bus has the following code that defines it as the root of the connection graph of Complex Power Port's frequency.

Connections.root (epp.f);

The PV Bus is defined as Connections. potentialRoot for the frequency of the 
Complex Power Port. These two functions are part of the Modelica language. Slack and PV busses are separate boundary models. For a PQ Bus, it is possible to either choose a simple boundary model or the load model with voltage and frequency dependency of active and reactive power. The frequency is part of the Complex Power Port due to the frequency dependency of the load.

When performing load flow calculations, the nodal voltages are determined. Because unknown quantities are initialized by zero, all grid busses need to be represented by one of these bus models. Even if no generation or consumption is connected to a bus, a boundary model must be chosen in order to prevent division by zero and to speed up the initialization.

The main linking component is the transmission line. Transmission lines are modeled using the Pi-network representation which is valid for electrically short transmission lines up to a length of approximately 150 $\mathrm{km}$.

For modeling the behavior of a transmission line, the $\mathrm{n}$-port representation is a powerful way to build its equations. When using admittance parameters in form of an admittance matrix $[\underline{Y}]$, the transmission line with its physical parameters Resistance $R$, Inductance $L$, Capacitance $C$ and angular frequency $\omega$ can be represented by the following equations.

$$
\begin{gathered}
\left(\underline{I_{1}}\right)=[\underline{Y}] \cdot\left(\frac{V_{1}}{\underline{V}_{2}}\right) \\
{[\underline{Y}]=\left[\begin{array}{cc}
\frac{1}{R+j \omega L}+\frac{j \omega C}{2} & \frac{-1}{R+j \omega L} \\
\frac{-1}{R+j \omega L} & \frac{1}{R+j \omega L}+\frac{j \omega C}{2}
\end{array}\right]} \\
\underline{S}_{i}=P_{i}+j Q_{i}=\underline{V}_{i} \cdot \underline{I}^{*}
\end{gathered}
$$

The port $i$ has the voltage $\underline{V}_{i}$, the current $\underline{I}_{i}$, the complex power $\underline{S}_{i}$, the active power $P_{i}$ and the reactive power $Q_{i}$. The power quantities are balanced powers. In the TransiEnt Library, the convention is that inflowing powers are positive and outflowing powers are negative. Consequently, generated power is assumed to be negative and consumed power to be positive at electrical connectors. With these equations, it is possible to describe the transmission line in a numerically efficient way that allows modeling of networks with up to hundreds of busses. Transmission lines can be parametrized by given values from literature or customized values. The TransiEnt Library additionally offers transformer models. Hence, electric networks with several voltage levels can be modeled and simulated.

Because of the overdetermined connector mentioned in section 2.1, the linking components must be defined as Connections.branch for the frequency of the Complex Power Port for the elimination of the surplus equation.

The load flow calculation function which has been implemented in the TransiEnt Library has been successfully validated with the commercial software tool NEPLAN® $(\mathrm{ABB}, 2016)$.

\subsection{Dynamic Components}

The dominant dynamic components in electric grids that are not fed by inverters are synchronous generators. Their behavior can be modeled in different levels of detail. Regarding the Complex Power Port, the simplest way to model a generator is to couple the active power with the frequency. The voltage is usually controlled and therefore set to a fixed desired value and the reactive power is calculated. The dynamic behavior is represented by the mechanical swing equation.

$$
\ddot{\phi}=\dot{\omega}=\frac{\omega_{0}}{T_{A}} \cdot \frac{P_{m e c h}-P_{e l}}{S_{r G}}
$$

The generator and the turbine rotate at the angular frequency $\omega$ that has the nominal value $\omega_{0}$. The angle $\phi$ denotes the machine's rotor wheel angle and the inertia is described by the time constant $T_{A}$. The generator which has the rated apparent power $S_{r G}$ is driven by the mechanical power $P_{\text {mech }}$ and generates the electric power $P_{e l}$. In the TransiEnt Library, the inertia of the generator and turbine is described with an additional component model for an inertia. Generator models are offered in a slack as well as in a PV bus version. The slack version fixes the voltage angle and therefore the voltage angle reference for the quasi-stationary consideration of the electric grid. The user can switch between both versions by setting a Boolean parameter.

For a higher level of detail, the coupling between the generator voltage magnitude and the active as well as reactive power is considered. In general, synchronous generators are typically modeled according to the Two Axis Method (Arrillaga Arnold 1990, Milano 2010). With the Two Axis Method, it is possible to describe the non-symmetric design of the magnet wheel. Only cylindrical rotor machines, which underlie slow dynamic changes, can be modeled using the synchronous reactance of just the direct axis which corresponds to a single-phase schematic as shown in Figure 2. 


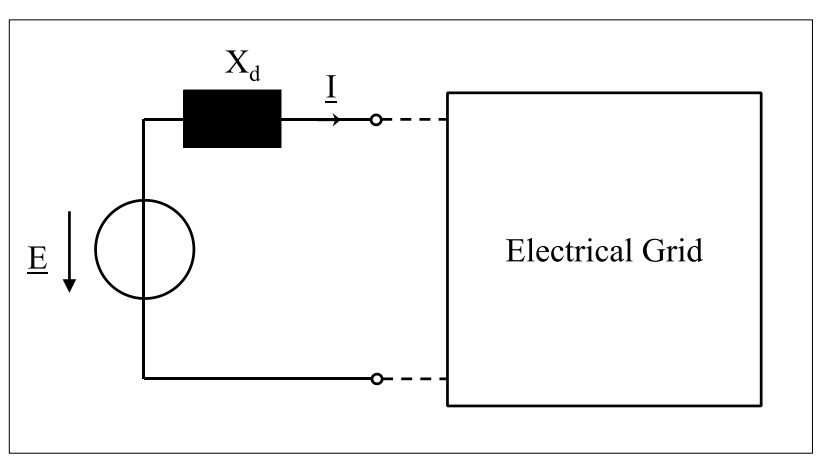

Figure 2. Steady-state model of synchronous generator as electric circuit with alternating voltage source $\underline{E}$ and synchronous reactance $X_{d}$.

The Two Axis Method is based on the Park Transformation and not explained in detail (Park, 1929).

Models with this coupling between voltage magnitude and the two forms of electric power need a voltage controller for operation in electric grids. Applied to the model in Figure 2, the voltage controller determines a value for the excitation current which leads to a voltage $\underline{E}$ to obtain the desired nominal voltage at the generator terminals. Models for voltage controllers are combined with excitation system models. These combined models are mainly linear time-invariant (LTI) systems represented as transfer functions in feedback loops. The Institute of Electrical and Electronics Engineers (IEEE) recommends different types of such models (IEEE, 2016). The TransiEnt Library offers DC and AC excitation system models in accordance with the IEEE recommendations.

Although the simulations in the ResiliEntEE project will be done with a single overall system frequency, the TransiEnt Library has the feature to compute different frequencies for generators. It is possible to activate a distinct frequency for every PV bus generator by a Boolean parameter. This feature is implemented by the following equation.

$$
\frac{d \theta_{p v}}{d t}=\omega_{p v}-\omega_{\text {slack }}
$$

The slack generator operates with the angular frequency $\omega_{\text {slack }}$ while the PV bus generator operates with $\omega_{p v}$ and has the polar wheel angle $\theta_{p v}$. The polar wheel angle of the slack bus is set to zero when using more than one frequency. Different frequencies in electric networks allow differently oscillating generators and thus power oscillations in large electric networks. Models for Power System Stabilizers (PSS) to damp power oscillations can be implemented when using this feature. With the consideration of more than one frequency, it becomes necessary to determine a frequency that is used as the overall grid frequency at different places in the network for dynamic loads and other models instead of the slack generator's frequency. This problem has not yet been faced by the TransiEnt
Library. Currently, this topic is worked at TU Ilmenau by combining phasor-based and electromagnetic transient (EMT) simulation to a hybrid simulation model which can be assumed as an approach for this problem (Jiang et al, 2018).

In existing models from the TransiEnt Library like power plant models, Modelica's modularity allows to define generator models, power boundaries and connectors as replaceable components. This allows the reuse of proven models from the non-electrical sectors. In addition, the replaceable components permit a variation of the model's level of detail.

\section{Simulated Examples}

To demonstrate the new features of the TransiEnt Library, some example system models are presented in this section.

\subsection{Steady-State Example}

First, there is a steady-state example for demonstrating the performance of load flow calculations. A test grid for Northern Germany has been created and implemented for performance analyses.

This Modelica model consists of three submodels. One model represents the electric high voltage grid of Mecklenburg-West Pomerania, another one SchleswigHolstein and Hamburg. The third sub model contains the high voltage grid of Lower Saxony and Bremen. Using the transformer models mentioned in section 2.2, it is possible to represent the three voltage levels $380 \mathrm{kV}$, $220 \mathrm{kV}$ and $110 \mathrm{kV}$. The topology is established manually from data published by the Transmission System Operators (TSO) (TenneT TSO GmbH, 2018; 50Hertz Transmission $\mathrm{GmbH}, 2018$ ). The model is only capable of representing one switching status of the real grid. Figure 3 shows the Schleswig-Holstein and Hamburg part of the model. 


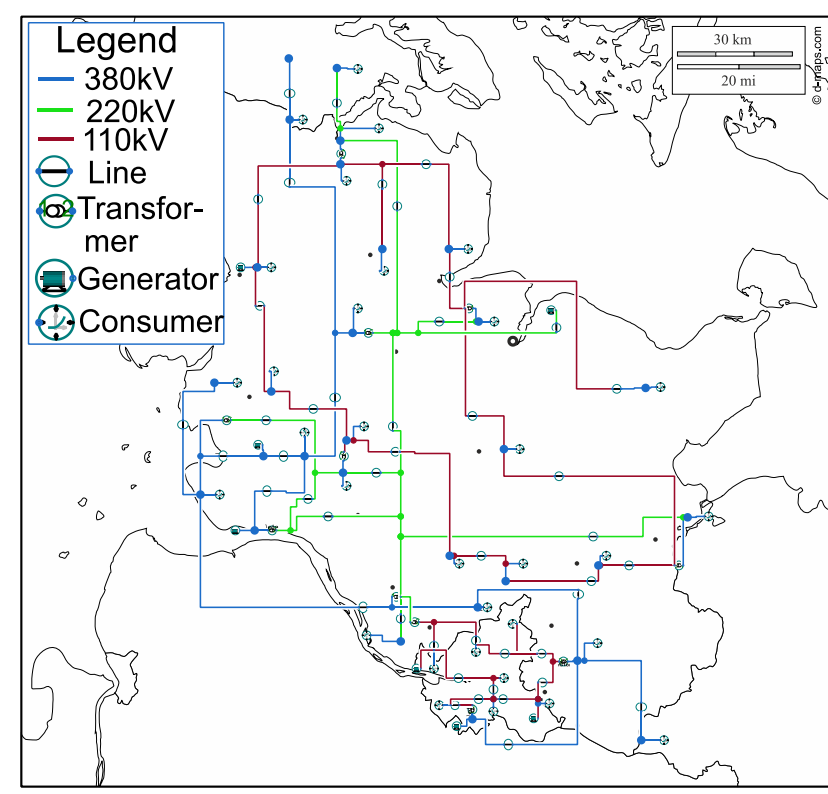

Figure 3. Model of the electric power grid of SchleswigHolstein and Hamburg in Dymola (d-maps, 2018).

Three scenarios are considered. The first scenario is the hypothetical operation of the Northern German grid as an electric island grid. The second scenario represents power transit from the direction of Denmark into the direction of the Netherlands. The third scenario is the so called Dunkelflaute scenario that assumes minimal wind and photovoltaic generation as well has high consumption. Especially, the third scenario shows a high use of the transmission line capacities which can be seen as an indication of congestion. Detailed results can be provided by the authors on request.

\subsection{Dynamic Example - Only Electrical}

The next model shows how dynamic simulations of electric power systems can be performed with the TransiEnt Library. This example covers only electric and some necessary mechanical components. It is purely theoretical and represents a grid with four generators supplying a load variation in active and reactive power. The example is used as a reference model in the TransiEnt Library for the test of the component models. The model size and the short time horizon allows to choose the most advanced generator model with Two Axis description and differential equations describing the magnetic effects. This does not increase the computational time significantly in comparison to generator models with more simplified equations. Figure 4 shows an excerpt of this simulation model. The turbine is modeled as a first order time delay and a mechanical boundary. The turbine is actuated by a linear system of the same type which represents the measurement delay and the proportional controller. The inertia of the whole rotating system is represented in an inertia model. The generator model is coupled to the electric grid by a transmission line and a transformer. A standard DC voltage controller is used. The bus between transmission line and transformer is initialized by a power boundary.

Figure 5 is an exemplary plot from this simulation and shows the reaction of the overall grid frequency to a load step. It shows a decrease in frequency because only primary control (proportional control) and no secondary control (integral control) is applied in this example. The bus voltages in this example are also variable and an example bus voltage on the $380 \mathrm{kV}$ level shows the time behavior in Figure 6. The voltage controller neither has an integral behavior. Consequently, the nominal voltage

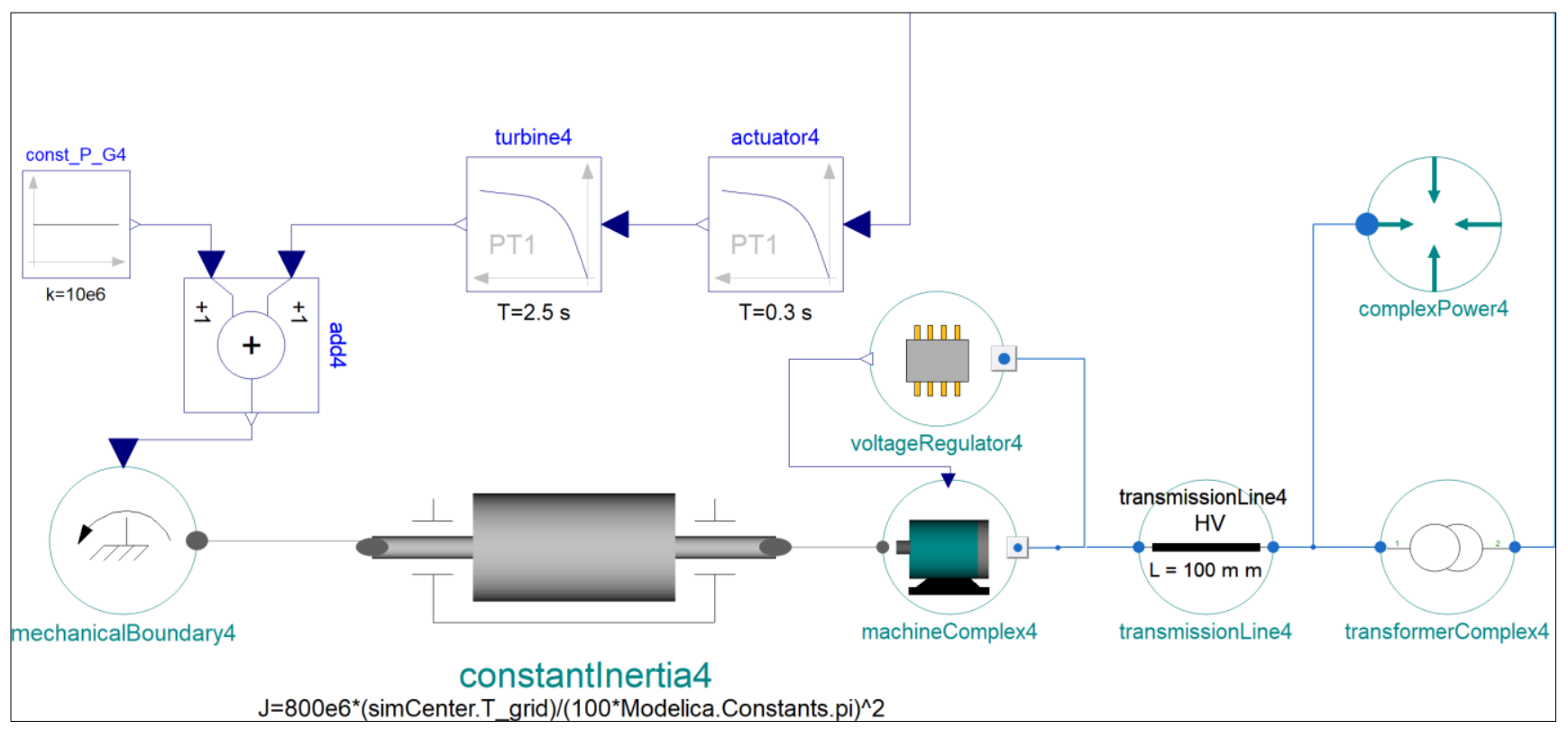

Figure 4. Excerpt of dynamic simulation model with mainly electric components. 
cannot be reached in steady-state before and after the load step.

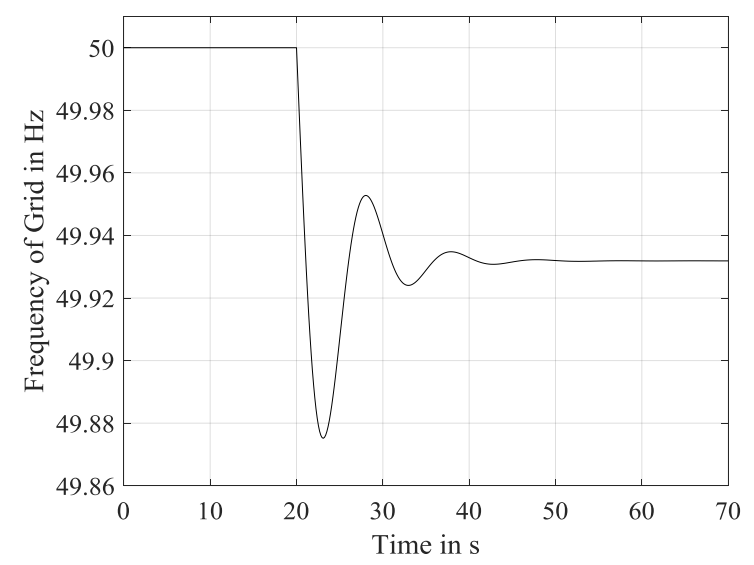

Figure 5. Time plot of grid frequency in dynamic example model.

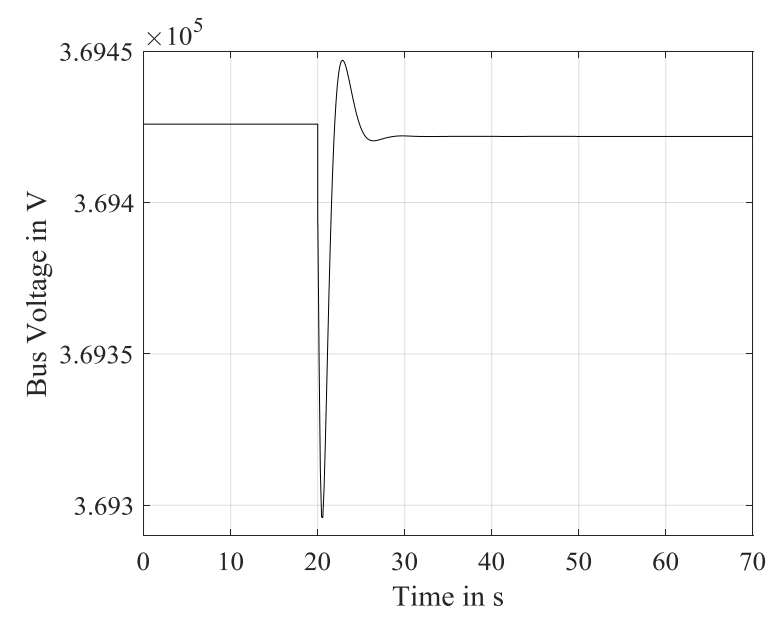

Figure 6. Time plot of bus voltage on the $380 \mathrm{kV}$ level.

\subsection{Dynamic Example - IES}

The third example should give an outlook to further planned simulations within the ResiliEntEE project. In these simulations, the components of all three sectors electricity, gas and heat constitute one simulation model that is simulated without the drawbacks of Cosimulation. Co-simulations typically need interfaces between different partial simulations. These interfaces handicap physical constraints such as mass and energy conservation and reduce the numerical efficiency.

Figure 7 shows the implemented dynamic system model. The model consists of two conventional power plants, a wind park as a renewable generation plant and a CHP plant. A natural gas grid is also part of the simulation model. For the heat sector, the CHP plant supplies a heat consumer. The feed-in-station feeds hydrogen into the gas grid when an excess of renewable energy generation in the right subpart of the model occurs. A battery storage is used to demonstrate some flexibility and is implemented as a voltage controlled device which sets the bus voltage to a fixed value. This sufficiently models the behavior of a power electronic inverter for the given time resolution.

When performing small parameter-variations, the overall system behavior changes noticeably, showing the high non-linearity of the system model. The model shows that a suitable operation management is necessary for IES. Especially, the storages must be controlled to have a beneficial effect on the electric grid. It can be shown that the battery storage helps to hold the electric grid frequency in the desired band of $\pm 50 \mathrm{mHz}$. This is done by a simple frequency control of the storage input and output power with a proportional controller. The behavior can thus be considered as an additional primary control by the battery storage. It can also be shown that the voltage control is important for setting the bus voltages to appropriate values to minimize transmission line losses and to prevent the overload of transmission lines. An insufficient choice leads to an overload of TransmissionLine and TransmissionLine 4 in the simulation.

\section{Summary and Outlook}

In this paper, the extension of the freely available TransiEnt Library for modeling IES with the method of dynamic simulation is shown. Compared to the models used until the end of the TransiEnt.EE research project, the new electrical models allow much more detailed dynamic modeling and analysis of electric power grids. Load flow calculations can be performed. The stability of the electric grid can be analyzed by considering frequency, voltage and angle stability. This is enabled by new models based on a new connector that allows interconnected networks without overdetermined DAE. Numerically efficient transmission line, transformer and generator models are provided in the TransiEnt Library. Generator models allow different levels of detail in dynamic modeling of the electric grid, starting with simple models that only regard active-power-frequency behavior up to models with excitation systems, Two Axis Method based equations and distinct frequencies. The high modularity of Modelica allows the simple adaption of existing models as well as the extension of existing models.

As already mentioned, the TransiEnt Library can be extended within the given framework. As extension, models for On-Load Tap Changer (OLTC), Flexible Alternating Current Transmission Systems (FACTS) and High Voltage Direct Current (HVDC) transmission are possible. The main goal is the simulation of a representative coupled system of Northern Germany. A strategy for the system configuration has already been considered and research for designing this complex simulation model is currently done. The idea is the separation of the complete system model into regional subsystems. In the ResiliEntEE project, this method is called Superstructure. Parallel to this, it is investigated which stability phenomena should be regarded in the 


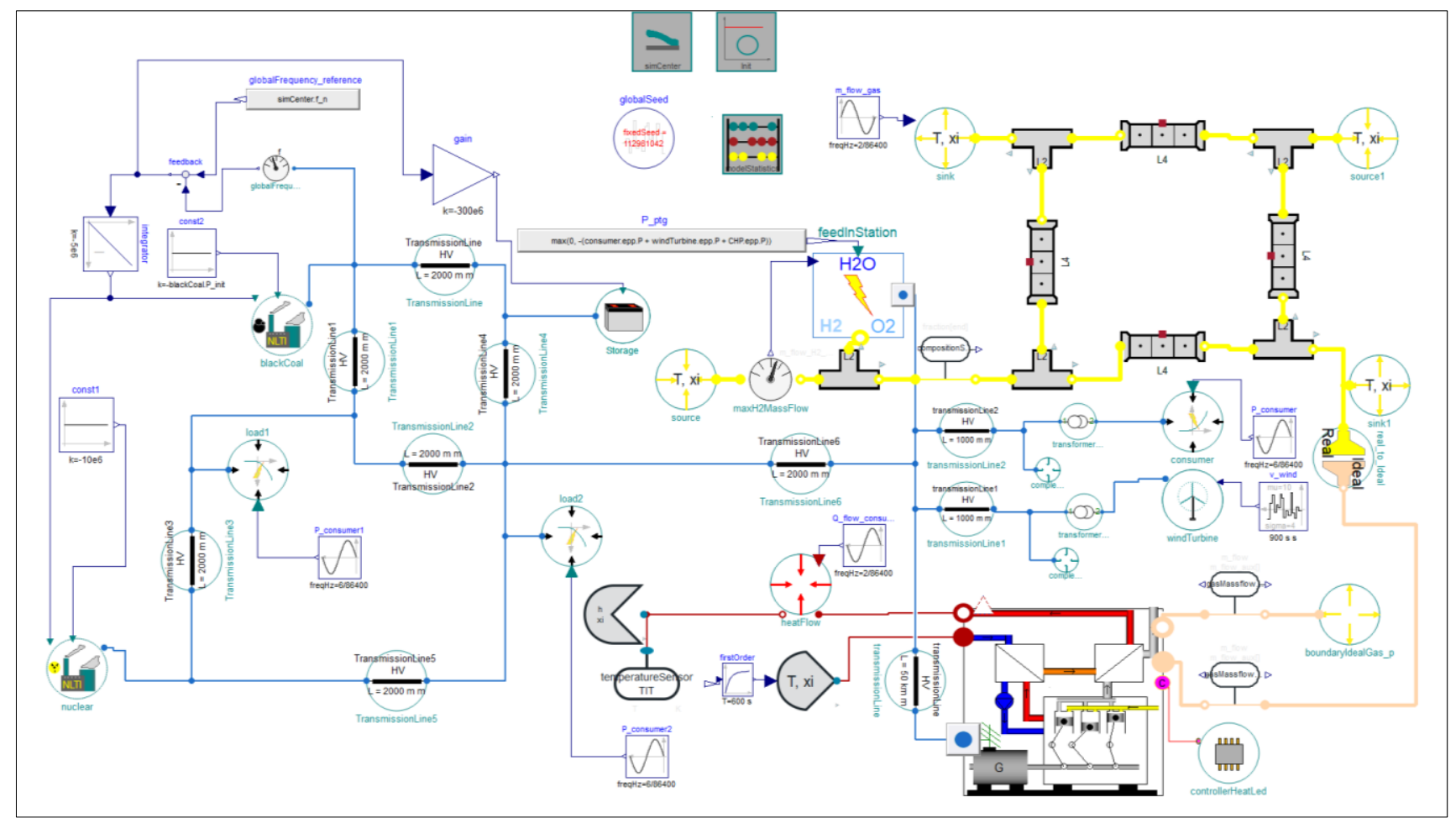

Figure 7. Example model for all three energy sectors within one simulation model in Dymola

electric grid for the chosen time and scenario horizon in order to investigate the overall system resilience.

The introduced features will be part of the upcoming release of the TransiEnt Library on the project website (University of Technology Hamburg, 2018).

\section{Acknowledgements}

The authors greatly acknowledge the funding from the German Federal Ministry of Economic Affairs and Energy for the project "ResiliEntEE - Resilience of coupled energy networks with a high share of renewable energies" (ResilientEE- Resilienz gekoppelter Energienetze mit hohem Anteil Erneuerbarer Energien, project number: 03ET4048).

\section{References}

ABB AG: NEPLAN® (Version V557). Available at: https://www.neplan.ch/neplanproduct/electricity, Zürich, Switzerland, 2016

L. Andresen, P. Dubucq, R. Peniche, G. Ackermann, A. Kather, G. Schmitz: Status of the TransiEnt Library: TransiEnt Simulation of Coupled Energy Networks with High Share of Renewable Energy. Proceedings of the 11th International Modelica Conference, Versailles, France, 2015.

L. Andresen, P. Dubucq, R. Peniche, G. Ackermann, A. Kather, G. Schmitz. Transientes Verhalten gekoppelter Energienetze mit hohem Anteil Erneuerbarer Energien. Abschlussbericht. Technische Informationsbibliothek, Hannover, Germany, 2017. doi: 10.2314/GBV:1002659345

J. Arrillaga, C. P. Arnold: Computer Analysis of Power Systems. John Wiley \& Sons Ltd, Chichester, United Kingdom, 1990 d-maps: Schleswig-Holstein (Deutschland) Grenzen (weiß). Available at: https://dmaps.com/carte.php?num_car=6499\&lang=de, Trets, France, 2018

Dassault Systèmes: Dymola ${ }^{\circledR}$ (Version 2019). Available at: https://www.3ds.com/de/produkte-undservices/catia/produkte/dymola/, Vélizy-Villacoublay, France, 2018

Hamburg University of Technology: TransiEnt Library. Available at: https://www.tuhh.de/transient-ee/, Hamburg, Germany, 2018

German Federal Ministry of Justice und Consumer Protection: Gesetz für den Ausbau erneuerbarer Energien (EEG). Available at: https://www.gesetze-iminternet.de/eeg_2014/, Berlin, Germany, 2017

IEEE Power and Energy Society: IEEE Recommended Practice for Excitation System Models for Power System Stability Studies. The Institute of Electrical and Electronics Engineers, Inc., New York, United States of America, 2016

T. Jiang, X. Song, S. Schlegel, D. Westermann: HybridsSimulation using eMEGASIM and ePHASORSIM for Converter Dominated Distribution Grid. NEIS Proceedings, Hamburg, Germany, 2018

F. Milano: Power System Modelling and Scripting. Springer, London, United Kingdom, 2010

Modelica Association: Modelica ${ }^{\circledR}$ - A Unified ObjectOriented Language for Systems Modeling — Language Specification. Version 3.4, Linköping, Sweden, 2017

R. H. Park: Two Reaction Theory of Synchronous Machines - Part I. AIEE Transactions, Vol 48, pp. 716-727, 1929

TenneT TSO GmbH: Statisches Netzmodell. Available at: https://www.tennettso.de/site/Transparenz/veroeffentlichu 
ngen/statisches-netzmodell/statisches-netzmodell,

Bayreuth, Germany, 2018

50Hertz Transmission GmbH: Statisches Netzmodell. Available at: https://www.50hertz.com/de/Anschluss-undZugang/Europaeischer-Stromhandel-undEngpassmanagement/Statisches-Netzmodell, Berlin, Germany, 2018 
